\title{
ON THE STABILITY OF SOLUTIONS OF IMPULSIVE NONLINEAR PARABOLIC EQUATIONS
}

\author{
Drumi BaINOV ${ }^{1}$ AND EMIL MinChEV ${ }^{1}$
}

\begin{abstract}
Stability and asymptotic stability of the solutions of impulsive nonlinear parabolic equations are studied via the method of differential inequalities.
\end{abstract}

Résumé. La stabilité et le comportement asymptotique de solutions d'équations paraboliques nonlinéaires impulsives sont étudiés via la méthode des inégalités différentielles.

AMS Subject Classification. 35B35.

Received: June 2, 1997. Revised: January 5, 1998.

\section{INTRODUCTION}

The theory of the impulsive ordinary differential equations underwent rapid development in the recent years $[1,3,7-9,11]$. The impulsive differential equations are adequate apparatus for mathematical simulation of many processes and phenomena in nature which are characterized by the fact that the system parameters are subject to short-term perturbations in time.

However, taking more factors into account leads to the development of the theory of impulsive partial differential equations. This theory marked its beginning with the paper [10]. The impulsive PDE provide natural framework for mathematical simulation of numerous processes and phenomena in theoretical physics, population dynamics, bio-technologies, chemistry, impulse technique and economics. We would like to point out the applications of the impulsive PDE in the quantum mechanics. In 1992 it was introduced a model of impulsive moving mirror $[13,14]$ presented by the apparatus of the impulsive PDE. For the present state of the theory of impulsive PDE we refer the reader to the monograph [2] and to the survey papers $[4-6,12]$.

In the present paper we study the stability and the asymptotic stability of the solutions of impulsive nonlinear parabolic equations via the method of differential inequalities.

\section{Preliminary notes}

Let $\Omega \subset \mathbb{R}^{n}$ be a bounded domain with a smooth boundary $\partial \Omega$ and $\bar{\Omega}=\Omega \cup \partial \Omega$. Suppose that

$$
0=x_{0}<x_{1}<x_{2}<\ldots<x_{k}<\ldots
$$

are given numbers such that $\lim _{k \rightarrow \infty} x_{k}=+\infty$.

Keywords and phrases. Stability of solutions, impulsive nonlinear parabolic equations.

${ }^{1}$ Medical University of Sofia, P.O. Box 45, Sofia 1504, Bulgaria. 
Define

$$
\begin{gathered}
E=\left\{(x, y) \in \mathbb{R}^{1+n}: \quad x \in \mathbb{R}_{+}, \quad y \in \bar{\Omega}\right\}, \quad \mathbb{R}_{+}=[0,+\infty), \\
\Gamma_{k}=\left\{(x, y) \in E: \quad x \in\left(x_{k}, x_{k+1}\right), \quad y \in \Omega\right\}, \quad k=0,1, \ldots, \quad \Gamma=\bigcup_{k=0}^{\infty} \Gamma_{k}, \\
B_{k}=\left\{(x, y) \in E: \quad x \in\left(x_{k}, x_{k+1}\right), \quad y \in \partial \Omega\right\}, \quad k=0,1, \ldots
\end{gathered}
$$

Let $C_{i m p}[E, \mathbb{R}]$ be the class of all functions $u: E \rightarrow \mathbb{R}$ such that:

(i) The functions $\left.u\right|_{\Gamma_{k} \cup B_{k}}, k=0,1, \ldots$, are continuous.

(ii) For each $k, k=1,2, \ldots, x=x_{k}$, there exists the limit

$$
\lim _{\substack{(s, q) \rightarrow(x, y) \\ s<x}} u(s, q)=u\left(x^{-}, y\right), \quad y \in \bar{\Omega} .
$$

(iii) For each $k, k=0,1, \ldots, x=x_{k}$, there exists the limit

$$
\lim _{\substack{(s, q) \rightarrow(x, y) \\ s>x}} u(s, q)=u\left(x^{+}, y\right), \quad y \in \bar{\Omega}
$$

and $u(x, y)=u\left(x^{+}, y\right), y \in \bar{\Omega}$.

Denote by $M[n]$ the class of all matrices $A=\left[a_{i j}\right]_{1 \leq i, j \leq n}$ with real entries. Let $f: \Gamma \times \mathbb{R} \times \mathbb{R}^{n} \times M[n] \rightarrow \mathbb{R}$, $\varphi: \mathbb{R}_{+} \times \partial \Omega \rightarrow \mathbb{R}, u_{0}: \bar{\Omega} \rightarrow \mathbb{R}, g:\left\{x_{k}\right\}_{k=1}^{\infty} \times \bar{\Omega} \times \mathbb{R} \rightarrow \mathbb{R}$ be given functions.

Consider the initial-boundary value problem (IBVP):

$$
\begin{aligned}
u_{x}(x, y) & =f\left(x, y, u(x, y), u_{y}(x, y), u_{y y}(x, y)\right), \quad(x, y) \in \Gamma, \\
u(0, y) & =u_{0}(y), \quad y \in \bar{\Omega}, \\
u(x, y) & =\varphi(x, y), \quad(x, y) \in \mathbb{R}_{+} \times \partial \Omega, \\
u\left(x_{k}, y\right) & =u\left(x_{k}^{-}, y\right)+g\left(x_{k}, y, u\left(x_{k}^{-}, y\right)\right), \quad y \in \bar{\Omega}, \quad k=1,2, \ldots,
\end{aligned}
$$

where $u_{y}(x, y)=\left(u_{y_{1}}(x, y), \ldots, u_{y_{n}}(x, y)\right), u_{y y}(x, y)=\left[u_{y_{i} y_{j}}(x, y)\right]_{1 \leq i, j \leq n}$.

Definition 1. A function $u: E \rightarrow \mathbb{R}$ is said to be a solution of the IBVP (1-4) if:

(i) $u \in C_{i m p}[E, \mathbb{R}]$, there exist continuous partial derivatives $u_{x}(x, y), u_{y}(x, y), u_{y y}(x, y)$ for $(x, y) \in \Gamma$ and $u$ satisfies (1) on $\Gamma$.

(ii) $u$ satisfies $(2-4)$.

Definition 2. The function $f: \Gamma \times \mathbb{R} \times \mathbb{R}^{n} \times M[n] \rightarrow \mathbb{R}$ is said to be elliptic at $\Gamma$ if for each point $(x, y) \in \Gamma$ and any $Q, S \in M[n]$ the quadratic form

$$
\sum_{i, j=1}^{n}\left(Q_{i j}-S_{i j}\right) \lambda_{i} \lambda_{j} \leq 0
$$

for arbitrary vector $\lambda \in \mathbb{R}^{n}$ implies

where $(u, P) \in \mathbb{R} \times \mathbb{R}^{n}$.

$$
f(x, y, u, P, Q) \leq f(x, y, u, P, S)
$$

We adopt the following definitions of stability:

Definition 3. The trivial solution $u(x, y) \equiv 0,(x, y) \in E$ of the IBVP (1-4) is said to be stable if for every $\varepsilon>0$ there exists $\delta=\delta(\varepsilon)>0$ such that $\left|u_{0}(y)\right|<\delta, y \in \bar{\Omega}$ and $|\varphi(x, y)|<\delta,(x, y) \in \mathbb{R}_{+} \times \partial \Omega$ imply $|u(x, y)|<\varepsilon$ on $E$. 
Definition 4. The trivial solution $u(x, y) \equiv 0,(x, y) \in E$ of the IBVP (1-4) is said to be asymptotically stable if:

(i) It is stable.

(ii) There exists a positive number $\delta_{0}$ such that to every $\varepsilon>0$ there corresponds $X(\varepsilon)$ such that $\left|u_{0}(y)\right|<\delta_{0}$, $y \in \bar{\Omega}$ and $|\varphi(x, y)|<\delta_{0},(x, y) \in \mathbb{R}_{+} \times \partial \Omega$ imply $|u(x, y)|<\varepsilon$ for $x \geq X(\varepsilon), y \in \bar{\Omega}$.

\section{MAin RESULTS}

Theorem 1. Let the following conditions hold:

1. The function $f$ is elliptic at $\Gamma$.

2. There exists a function $\tilde{f} \in C\left(\left(\mathbb{R}_{+} \backslash\left\{x_{k}\right\}_{k=1}^{\infty}\right) \times \mathbb{R}, \mathbb{R}\right)$ such that

$$
f(x, y, p, 0,0) \leq \widetilde{f}(x, p)
$$

for $(x, y) \in \Gamma, p \in \mathbb{R}$.

3. There exists a function $\left.\widetilde{g} \in C\left(\left\{x_{k}\right\}_{k=1}^{\infty}\right) \times \mathbb{R}, \mathbb{R}\right)$ such that

$$
g\left(x_{k}, y, p\right) \leq \widetilde{g}\left(x_{k}, p\right)
$$

$y \in \bar{\Omega}, p \in \mathbb{R}, k=1,2, \ldots$

4. The function $p+\widetilde{g}\left(x_{k}, p\right)$ is nondecreasing on $\mathbb{R}$ for each $k, k=1,2, \ldots$

5. There exists a function $\gamma(x)$ which is a maximal solution of the problem

$$
\begin{aligned}
\gamma^{\prime}(x) & =\widetilde{f}(x, \gamma(x)), \quad x \neq x_{k}, \\
\gamma(0) & =\gamma_{0}, \\
\gamma\left(x_{k}\right) & =\gamma\left(x_{k}^{-}\right)+\widetilde{g}\left(x_{k}, \gamma\left(x_{k}^{-}\right)\right), \quad k=1,2, \ldots,
\end{aligned}
$$

where

$$
\begin{gathered}
u_{0}(y) \leq \gamma_{0}, \quad y \in \bar{\Omega} \\
\varphi(x, y) \leq \gamma(x), \quad(x, y) \in \mathbb{R}_{+} \times \partial \Omega
\end{gathered}
$$

Then for any solution $u$ of the IBVP (1-4) we have that

$$
u(x, y) \leq \gamma(x) \quad \text { on } \quad E .
$$

Proof. Let $T_{0}>0, E_{T_{0}}=\left[0, T_{0}\right] \times \bar{\Omega}$ and there exist a positive integer $m$ such that $x_{m}<T_{0}<x_{m+1}$. We prove that

$$
u(x, y) \leq \gamma(x) \quad \text { on } \quad E_{T_{0}} .
$$

There exists $\varepsilon_{0}>0$ such that for $0<\varepsilon<\varepsilon_{0}$, a solution $\gamma(\cdot ; \varepsilon)$ of the problem

$$
\begin{aligned}
\gamma^{\prime}(x ; \varepsilon) & =\widetilde{f}(x, \gamma(x ; \varepsilon))+\varepsilon, \quad x \neq x_{k}, \\
\gamma(0 ; \varepsilon) & =\gamma(0)+\varepsilon \\
\gamma\left(x_{k} ; \varepsilon\right) & =\gamma\left(x_{k}^{-} ; \varepsilon\right)+\widetilde{g}\left(x_{k}, \gamma\left(x_{k}^{-} ; \varepsilon\right)\right)+\varepsilon, \quad k=1,2, \ldots, m,
\end{aligned}
$$


is defined on $\left[0, T_{0}\right]$ and $\lim _{\varepsilon \rightarrow 0} \gamma(x ; \varepsilon)=\gamma(x)$, uniformly on $\left[0, T_{0}\right]$. We prove that

$$
u(x, y)<\gamma(x ; \varepsilon) \quad \text { on } \quad E_{T_{0}} .
$$

Suppose that (13) is not true. Then the set $Z=\left\{x \in\left[0, T_{0}\right]:\right.$ there exists $y \in \bar{\Omega}$ such that $\left.u(x, y) \geq \gamma(x ; \varepsilon)\right\}$ is non-empty. Defining $\widetilde{x}=\inf Z$. It follows from (8) that $\widetilde{x}>0$ and there exists a point $\widetilde{y} \in \bar{\Omega}$ such that

$$
\begin{aligned}
& u(x, y)<\gamma(x ; \varepsilon), \quad(x, y) \in[0, \widetilde{x}) \times \bar{\Omega}, \\
& u(\widetilde{x}, \widetilde{y})=\gamma(\widetilde{x} ; \varepsilon) .
\end{aligned}
$$

There are three cases to be distinguished:

Case 1. $\widetilde{y} \in \partial \Omega$. Then in view of (9)

$$
u(\widetilde{x}, \widetilde{y})=\gamma(\widetilde{x} ; \varepsilon)>\gamma(\widetilde{x}) \geq \varphi(\widetilde{x}, \widetilde{y})
$$

and we get a contradiction.

Case 2. $(\widetilde{x}, \widetilde{y}) \in \Gamma$. Then we have

$$
\begin{aligned}
& u_{x}(\widetilde{x}, \widetilde{y}) \geq \gamma^{\prime}(\widetilde{x} ; \varepsilon), \quad u_{y}(\widetilde{x}, \widetilde{y})=0, \\
& \sum_{i, j=1}^{n} u_{y_{i} y_{j}}(\widetilde{x}, \widetilde{y}) \lambda_{i} \lambda_{j} \leq 0, \quad \lambda \in \mathbb{R}^{n} .
\end{aligned}
$$

From the ellipticity of $f$ and (5) we obtain

$$
\begin{aligned}
0 & \leq u_{x}(\widetilde{x}, \widetilde{y})-\gamma^{\prime}(\widetilde{x} ; \varepsilon) \\
& \leq f(\widetilde{x}, \widetilde{y}, u(\widetilde{x}, \widetilde{y}), 0,0)-\widetilde{f}(\widetilde{x}, \gamma(\widetilde{x} ; \varepsilon))-\varepsilon<0,
\end{aligned}
$$

which is a contradiction.

Case 3. $\widetilde{x}=x_{k}$ for some $k, 1 \leq k \leq m$. Then we obtain from (14) that

$$
\begin{gathered}
u\left(x_{k}^{-}, \widetilde{y}\right) \leq \gamma\left(x_{k}^{-} ; \varepsilon\right), \\
u\left(x_{k}, \widetilde{y}\right)=\gamma\left(x_{k} ; \varepsilon\right) .
\end{gathered}
$$

From (6), (15) and condition 4 of the theorem we conclude that

$$
\begin{aligned}
0 & =u\left(x_{k}, \widetilde{y}\right)-\gamma\left(x_{k} ; \varepsilon\right) \\
& =u\left(x_{k}^{-}, \widetilde{y}\right)+g\left(x_{k}, \widetilde{y}, u\left(x_{k}^{-}, \widetilde{y}\right)\right)-\gamma\left(x_{k}^{-} ; \varepsilon\right)-\widetilde{g}\left(x_{k}, \gamma\left(x_{k}^{-} ; \varepsilon\right)\right)-\varepsilon \\
& \leq u\left(x_{k}^{-}, \widetilde{y}\right)+\widetilde{g}\left(x_{k}, u\left(x_{k}^{-}, \widetilde{y}\right)\right)-\gamma\left(x_{k}^{-} ; \varepsilon\right)-\widetilde{g}\left(x_{k}, \gamma\left(x_{k}^{-} ; \varepsilon\right)\right)-\varepsilon<0,
\end{aligned}
$$

which is a contradiction.

Hence the set $Z$ is empty and (13) follows. Since $\lim _{\varepsilon \rightarrow 0} \gamma(x ; \varepsilon)=\gamma(x)$ uniformly on $\left[0, T_{0}\right]$ we conclude that

$$
u(x, y) \leq \gamma(x) \quad \text { on } \quad E_{T_{0}} .
$$

Since $T_{0}>0$ was arbitrary we get the estimate (10). 
Theorem 2. Let the following conditions hold:

1. Conditions 1-4 of Theorem 1 are fulfilled.

2. There exists a function $\gamma(x)$ which is a maximal solution of the problem (7), where

$$
\begin{gathered}
\left|u_{0}(y)\right| \leq \gamma_{0}, \quad y \in \bar{\Omega}, \\
|\varphi(x, y)| \leq \gamma(x), \quad(x, y) \in \mathbb{R}_{+} \times \partial \Omega .
\end{gathered}
$$

3. $f(x, y,-p,-q,-r)=-f(x, y, p, q, r)$ for $(x, y, p, q, r) \in \Gamma \times \mathbb{R} \times \mathbb{R}^{n} \times M[n]$.

4. $g\left(x_{k}, y,-p\right)=-g\left(x_{k}, y, p\right), y \in \bar{\Omega}, p \in \mathbb{R}, k=1,2, \ldots$

Then for any solution $u$ of the IBVP (1-4) we have that

$$
|u(x, y)| \leq \gamma(x) \quad \text { on } \quad E .
$$

Proof. The estimate

$$
u(x, y) \leq \gamma(x) \quad \text { on } \quad E
$$

follows from Theorem 1 . Now we will prove that

$$
-u(x, y) \leq \gamma(x) \quad \text { on } \quad E .
$$

It follows from conditions 3 and 4 of Theorem 2 that the function $-u(x, y)$ is a solution of the problem

$$
\begin{aligned}
u_{x}(x, y) & =f\left(x, y, u(x, y), u_{y}(x, y), u_{y y}(x, y)\right), \quad(x, y) \in \Gamma, \\
u(0, y) & =-u_{0}(y), \quad y \in \bar{\Omega}, \\
u(x, y) & =-\varphi(x, y), \quad(x, y) \in \mathbb{R}_{+} \times \partial \Omega, \\
u\left(x_{k}, y\right) & =u\left(x_{k}^{-}, y\right)+g\left(x_{k}, y, u\left(x_{k}^{-}, y\right)\right), \quad y \in \bar{\Omega}, \quad k=1,2, \ldots
\end{aligned}
$$

Having in mind the estimates (16) and (17), we conclude by Theorem 1 that the inequality (19) holds true.

Relations (18) and (19) prove the conclusion of the theorem.

Theorem 3. Let the following conditions hold:

1. Conditions 1, 3, 4 of Theorem 2 are fulfilled.

2. The maximal solution $\gamma(x)$ of the problem $(7)$ is defined on $\mathbb{R}_{+}$and it is nonnegative.

3. $f(x, y, 0,0,0)=0$ for $(x, y) \in \Gamma, g\left(x_{k}, y, 0\right)=0, y \in \bar{\Omega}, \widetilde{f}(x, 0)=0, x \in \mathbb{R}_{+} \backslash\left\{x_{k}\right\}_{k=1}^{\infty}, g\left(x_{k}, 0\right)=0$, $k=1,2, \ldots$

Then the stability or asymptotic stability of the trivial solution of the problem (7) implies stability or asymptotic stability of the trivial solution of the IBVP (1-4).

Proof. Suppose that the trivial solution of the problem (7) is stable. Then for every $\varepsilon>0$ there exists $\delta=\delta(\varepsilon)>0$ such that $\gamma_{0}<\delta$ implies $\gamma(x)<\varepsilon$ on $\mathbb{R}_{+}$. Then by Theorem 2 it follows that

$$
|u(x, y)| \leq \gamma(x) \quad \text { on } \quad E
$$

provided that

$$
\left|u_{0}(y)\right| \leq \gamma_{0}, \quad y \in \bar{\Omega}
$$


and

$$
|\varphi(x, y)| \leq \gamma(x), \quad(x, y) \in \mathbb{R}_{+} \times \partial \Omega .
$$

This proves the stability of the trivial solution of the IBVP (1-4).

Analogously it can be proved that the asymptotic stability of the trivial solution of (7) implies asymptotic stability of the trivial solution of the IBVP (1-4).

\section{Applications in the population Dynamics}

Particular interest for the mathematical biology is the special case of IBVP (1-4) when $f(x, y, p, q, r)=$ $\kappa r+p\left(a-b p^{2}\right), \kappa>0, a>0, b>0$ are constants and $\varphi(x, y) \equiv 0$. Then the IBVP (1-4) takes on the form

$$
\begin{aligned}
u_{x}(x, y) & =\kappa \Delta u(x, y)+u(x, y)\left(a-b u^{2}(x, y)\right), \quad(x, y) \in \Gamma, \\
u(0, y) & =u_{0}(y), \quad y \in \bar{\Omega} \\
u(x, y) & =0, \quad(x, y) \in \mathbb{R}_{+} \times \partial \Omega, \\
u\left(x_{k}, y\right) & =u\left(x_{k}^{-}, y\right)+g\left(x_{k}, y, u\left(x_{k}^{-}, y\right)\right), \quad y \in \bar{\Omega}, k=1,2, \ldots
\end{aligned}
$$

The IBVP (20-23) describes a single species population in a bounded environment. The function $u(x, y)$ represents the population density at the point $y \in \bar{\Omega}$ and time $x \geq 0$. Condition (23) describes instantaneous changes in the population density due to phenomena as: harvesting, disasters, immigration, emigration, etc.

Suppose that $g\left(x_{k}, y, p\right)=\beta_{k} p, \beta_{k}>-1, y \in \bar{\Omega}, k=1,2, \ldots$, and

$$
\prod_{s<x_{k}<x}\left(\frac{1}{1+\beta_{k}}\right) \geq L e^{-\beta(x-s)},
$$

where $L>0, \beta>0$ are constants. Let $\gamma_{0}=\max _{y \in \bar{\Omega}} u_{0}(y)$ and $u$ be a solution of the IBVP (20-23). Then we consider the problem

$$
\begin{aligned}
\gamma^{\prime}(x) & =\gamma(x)\left(a-b \gamma^{2}(x)\right), \quad x \neq x_{k}, \\
\gamma(0) & =\gamma_{0}, \\
\gamma\left(x_{k}\right) & =\gamma\left(x_{k}^{-}\right)+\beta_{k} \gamma\left(x_{k}^{-}\right), \quad k=1,2, \ldots
\end{aligned}
$$

We substitute $\rho(x)=\frac{1}{\gamma^{2}(x)}$ and obtain the problem

$$
\begin{aligned}
\rho^{\prime}(x) & =-2 a \rho(x)+2 b, \quad x \neq x_{k}, \\
\rho(0) & =\rho_{0}=\frac{1}{\gamma_{0}^{2}}, \\
\rho\left(x_{k}\right) & =\frac{1}{\left(1+\beta_{k}\right)^{2}} \rho\left(x_{k}^{-}\right), \quad k=1,2, \ldots
\end{aligned}
$$


Then we have

$$
\begin{aligned}
\rho(x) & =\rho_{0} \prod_{0<x_{k} \leq x}\left(\frac{1}{1+\beta_{k}}\right)^{2} e^{-2 a t}+\int_{0}^{x} \prod_{s<x_{k} \leq x}\left(\frac{1}{1+\beta_{k}}\right)^{2} e^{-2 a(x-s)} 2 b d s \\
& \geq L \rho_{0} e^{-2(a+\beta) x}+2 L b \int_{0}^{x} e^{-2 \beta(x-s)} e^{-2 a(x-s)} d s \\
& =\left(L \rho_{0}-\frac{L b}{a+\beta}\right) e^{-2(a+\beta) x}+\frac{L b}{a+\beta} .
\end{aligned}
$$

Therefore

and by Theorem 2 we conclude that

$$
\gamma(x) \leq\left[L\left(\frac{1}{\gamma_{0}^{2}}-\frac{b}{a+\beta}\right) e^{-2(a+\beta) x}+\frac{L b}{a+\beta}\right]^{-1 / 2},
$$

$$
|u(x, y)| \leq\left[L\left(\frac{1}{\gamma_{0}^{2}}-\frac{b}{a+\beta}\right) e^{-2(a+\beta) x}+\frac{L b}{a+\beta}\right]^{-1 / 2} .
$$

On the other hand, we have by Theorem 3 that the stability of the trivial solution of (24-26) implies stability of the trivial solution of IBVP (20-23).

The authors express their deep gratitude to the referee for his valuable advices and helpful suggestions. The present investigation was partially supported by the Bulgarian Ministry of Education and Science under grant MM-702.

\section{REFERENCES}

[1] D. Bainov and V. Covachev, Impulsive Differential Equations with a Small Parameter. World Scientific Publishers, Singapore (1994).

[2] D. Bainov, Z. Kamont and E. Minchev, Approximate Solutions of Impulsive Hyperbolic Equations. Academic Publishers, Calcutta (1996).

[3] D. Bainov and S. Kostadinov, Abstract Impulsive Differential Equations. Descartes Press, Koriyama (1996).

[4] D. Bainov and E. Minchev, Impulsive partial differential equations of first order - (I) Theorems on impulsive differential inequalities. J. Henan Univ. (Nat. Sci.) 25 (1995) 9-18.

[5] D. Bainov and E. Minchev, Impulsive partial differential equations of first order - (II) Stability of solutions and difference methods. J. Henan Univ. (Nat. Sci.) 26 (1996) 1-13.

[6] D. Bainov and E. Minchev, Trends in the theory of impulsive partial differential equations. Nonlinear World 3 (1996) $357-384$.

[7] D. Bainov and P. Simeonov, Systems with Impulse Effect, Stability, Theory and Applications. Ellis Horwood, Chichester (1989).

[8] D. Bainov and P. Simeonov, Theory of Impulsive Differential Equations: Asymptotic Properties of the Solutions. World Scientific Publishers, Singapore (1995).

[9] D. Bainov and P. Simeonov, Theory of Impulsive Differential Equations: Periodic Solutions and Applications. Longman, Harlow (1993).

[10] L.H. Erbe, H.I. Freedman, X.Z. Liu and J.H. Wu, Comparison principles for impulsive parabolic equations with applications to models of single species growth. J. Austral. Math. Soc., Ser. B 32 (1991) 382-400.

[11] V. Lakshmikantham, D. Bainov and P. Simeonov, Theory of Impulsive Differential Equations. World Scientific Publishers, Singapore (1989).

[12] E. Minchev and I.E. Okoroafor, Present state of the qualitative theory of the impulsive partial differential equations. Comm. Appl. Anal. 1 (1997) 351-369.

[13] G. Petrov, Impulsive moving mirror model and impulsive differential equations in Banach space. Communications of the Joint Institute for Nuclear Research, preprint E2-92-276, Dubna, Russia (1992).

[14] G. Petrov, Impulsive moving mirror model in a Schrödinger picture with impulse effect in a Banach space. Communications of the Joint Institute for Nuclear Research, preprint E2-92-272, Dubna, Russia (1992). 